\title{
Meta-análisis: Más evidencia a favor del uso de tiazidas en el manejo de la hipertensión arterial
}

Systematic review of antihypertensive therapies: Does the evidence assist in choosing a first- line drug? Wright JM, Lee $\mathrm{CH}$, Chambers GK. CMAJ 1999; 161: 25-32.

\section{Objetivo}

Evaluar la evidencia disponible sobre eficacia y efectividad de los tratamientos farmacológicos antihipertensivos comparados entre sí y contra placebo.

\section{Fuente y Selección de Datos}

Medline (1966-1997), Cochrane Library (hasta 1998), referencias sobre meta-análisis previos publicados entre 1980 y 1997

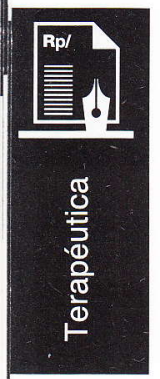

\section{Extracción de Datos}

Fueron seleccionados todos los ensayos clínicos controlados aleatorizados (ECCA) de al menos un año de seguimiento, que reportaran datos sobre morbimortalidad y con al menos una TA de $160 / 90 \mathrm{mmHg}$, cualquiera fuera su comorbilidad de base. Se incluyeron los estudios que compararan droga contra placebo y droga contra droga. Se reportó información sobre eficacia antihipertensiva, tolerancia e impacto sobre la morbimortalidad: muerte, accidente cerebro vascular (ACV), enfermedad coronaria (EC) incluyendo infarto fatal, no fatal y muerte súbita, eventos cardiovasculares totales (ECT) incluyendo ACV, EC, Insuficiencia Cardiaca (IC), y ruptura aneurismática. El análisis fue realizado agrupando a los estudios según fueran droga- placebo o drogadroga y según el tipo de fármaco.

\section{Resultados principales}

Fueron incluidos 23 ECCA que sumaron 50.853 pacientes.

\section{Estudios que compararon droga vs. droga:}

Las tiazidas fueron similares a los beta-bloqueantes (BB) en la reducción de eventos, pero en los ECT diferencia en el límite de la significancia estadística a su favor. Fueron superiores en tolerancia. Las tiazidas fueron similares a los bloqueantes cálcicos (BC) en reducción de eventos y tolerancia. Y los BC fueron similares a los inhibidores de la enzima de conversión (IECA) en la reducción de eventos pero fueron claramente mejor tolerados. (Tabla 1)

Tabla 1: Riesgos relativos de eventos y tolerancia en los ensayos que compararon droga-droga.

\begin{tabular}{|c|c|c|c|}
\hline & $\begin{array}{l}\text { Tiazidas vs. BB (5 ECCA) } \\
\text { RR (IC 95\%) }\end{array}$ & \begin{tabular}{|l} 
Tiazidas vs. BC (2 ECCA) \\
RR (IC 95\%)
\end{tabular} & $\begin{array}{l}\text { IECA vs. BC (1 ECCA) } \\
\text { RR (IC } 95 \%)\end{array}$ \\
\hline Muerte & $0.97 \quad(0.84-1.11)$ & $1.00(0.47-2.15)$ & $0.73(0.16-3.26)$ \\
\hline ACV & $0.84 \quad(0.65-1.08)$ & $0.64 \quad(0.25-1.64)$ & $0.39(0-12-1-24)$ \\
\hline EC & $0.91 \quad(0.78-1.07)$ & $1.00(0.50-1.99)$ & $4.90(0.23-10.1)$ \\
\hline ECT & $0.88 \quad(0.78-1.00)$ & $0.75 \quad(0.45-1.27)$ & $0.49(0.18-1.29)$ \\
\hline Abandono & $0.69+(0.63-0.76)$ & $0.91 \quad(0.62-1.33)$ & $3.31+(2.19-5.00)$ \\
\hline
\end{tabular}

Eficacia: las tiazidas fueron superiores a los BB y a los BC en la reducción de la TAS; y los $B C$ fueron superiores a los IECA. En la reducción de la $\mathrm{TAD}$ no hubo diferencias entre tiazidas, $\mathrm{BB}$ y $\mathrm{BC}$; sin embargo los BC fueron superiores a los IECA.

Estudios que compararon droga vs. placebo:

Las tiazidas en bajas dosis fueron ampliamente superiores al placebo en la reducción de eventos. Cuando las tiazidas fueron utilizadas en altas dosis no se observaron diferencias en la reducción de muerte y EC. Para los BB, ninguno de los eventos fue reducido en forma significativa y para los $\mathrm{BC}$ hubo diferencia significativa en la reducción de ACV y ECT, sin embargo no hubo prevención de muerte y EC en forma significativa. Tabla 2

Tabla 2: Riesgos relativos de eventos y NNT (ECT)en los ensayos que compararon drogas-placebo (seguimiento de 27 años).

\begin{tabular}{|c|c|c|c|c|}
\hline & $\begin{array}{l}\text { Tiazidas hajas dosis } \\
\text { (5 ECCA) } \\
\text { RR (IC 95\%) }\end{array}$ & $\begin{array}{l}\text { Tiazidas allas dosis } \\
\text { (11 ECCA) } \\
\text { RR (LC 95\%) }\end{array}$ & $\begin{array}{l}\text { BB (2 ECCA) } \\
\text { RR (LC } 95 \%)\end{array}$ & $\begin{array}{l}\text { BC (1 ECCA) } \\
\text { BR (IC 95\%) }\end{array}$ \\
\hline Muerte & $0.89+(0.82-0.99)$ & $0.90 \quad(0.76-1.05)$ & $1.01(0.88-1.15)$ & $0.86 \quad(0.68-1.09)$ \\
\hline $\mathrm{ACV}$ & $0.66+(0.56-0.79)$ & $0.47+(0.37-0.61)$ & $0.80(0.64-1.01)$ & $0.61+(0.43-0.87)$ \\
\hline$E C$ & $0.71+(0.60-0.84)$ & $1.00 \quad(0.84-1.19)$ & $0.92(0.78-1.10)$ & $0.76 \quad(0.54-1.07)$ \\
\hline ECT & $0.68+(0.62-0.75)$ & $0.72+(0.63-0.82)$ & $0.89(0.78-1.02)$ & $0.71+(0.57-0.87$ \\
\hline NNT & 18 & 67 & 142 & 42 \\
\hline
\end{tabular}

Eficacia: tanto para TAS como para TAD todas las drogas fueron claramente mejores que el placebo. Las tiazidas fueron mejores que todos los otros fármacos con una diferencia de $4-5 \mathrm{mmHg}$. No hubo diferencia entre las bajas y las altas dosis de tiazidas. Conclusiones

La evidencia sobre los beneficios de utilizar tiazidas en dosis bajas como drogas de primera línea, es robusta, tanto por eficacia antihipertensiva, por la reducción de eventos vasculares y por la tolerancia. No deben utilizarse tiazidas en dosis altas.

\section{Comentario}

Fuente de Financiamiento: British Columbia Ministry of Health y University of British Columbia.

No cabe duda de la eficacia antihipertensiva y del beneficio cardiovascular que proporcionan las tiazidas, constituyéndose en las drogas que gozan de la mejor calidad de evidencia. Varias guías de manejo, entre ellas el JNC VI, las recomiendan como drogas de primera línea en el manejo de la HTA. ${ }^{1}$ Dos meta- análisis previos arrojaron datos similares a cerca de su efectividad.2-3 A pesar de ello las tiazidas no son las drogas más usadas en la practica cotidiana en relación a los fármacos nuevos. Un elemento de radical importancia es el beneficio de su utilización en dosis bajas (12.5 mg. a $25 \mathrm{mg} /$ día) dado que: 1) Son tan eficaces como las dosis mas altas; 3 2) Disminuyen el riesgo coronario y las muertes totales (a diferencia de las dosis altas); 3) Presentan menores efectos adversos que las dosis altas. Los efectos metabólicos y el riesgo de arritmias son prácticamente inexistentes a dosis bajas. ${ }^{4}$ Una limitación de este meta-análisis, es que no discrimina grupos etarios ni comorbilidades previas, en el reporte de los resultados. En los ensayos droga-placebo del presente estudio, se reporta que las tiazidas redujeron eventos en forma estadísticamente significativa a diferencia de los BB. Sería erróneo concluir que las tiazidas son mejores que los BB basándose en estos resultados ya que estaría realizándose una comparación indirecta entre estudios cuyas poblaciones fueron diferentes (en edad y comorbilidad previa). Cuando los BB fueron comparados contra las tiazidas en 5 ECCA no hubo diferencias significativas entre ambos. Ya no es esperable, por razones obvias, que aparezcan nuevos estudios drogas vs. placebo. Sin embargo desde la publicación del presente meta-análisis hasta la actualidad aparecieron algunos estudios que compararon droga vs. droga y que aumentaron el caudal de evidencia sobre las drogas nuevas (IECA y BC de acción prolongada) mostrando que son similares, pero no superiores, a las drogas convencionales (tiazidas y BB) en la reducción de eventos cardiovasculares. El estudio ALLAHT (aun no publicado) aportará información muy relevante al respecto.

Mientras tanto, y hasta gozar de mayor y mejor calidad de evidencia, siempre que sea posible debiéramos intentar iniciar tratamiento antihipertensivo con tiazidas en pacientes sin otras comorbilidades, por su contundente eficacia, tolerancia y bajo costo. Esto último debe ser tenido en cuenta en vista de la magnitud de la HTA como problema sanitario y la necesidad de realizar intervenciones costo-efectivas.

1.- Joint National Committee on Prevention, Detection, Evaluation and Treatment of High Blood Pressure. The Sixth Report. Arch. Intern Med., Nov 24, 1997; 157:2413-2445. 2.- Collins R et al. Blood pressure, stroke, and coronary heart disease. Part 2: short- term reductions in blood pressure: overview of randomized drug trials in their epidemiological conext. Lancet 1990: 335: 827- 38

3.- Psaty BM, et al. Health outcomes associated with antihypertensive therapies used as first- line agents. A systematic review and meta-analysis JAMA 1997 Mar 5:277 (9). 739-45 4.- Flack JM, et al. Evidence for the efficacy of low-dose diuretic monotherapy. Am J Med 1996 Sep 30; 101 (3A): 53S-60S. 